\title{
Diseño e implementación de pruebas nacionales -ICFES-e internacionales -PISA- a partir del uso del aula virtual ${ }^{1}$
}

\author{
Andrea Carolina Álvarez \\ carolo3914@hotmail.com \\ Fernando Calderón \\ edward80204@hotmail.com \\ Nicolás Jaramillo \\ nicopinocho@hotmail.com \\ Adriana León \\ arianaleon_666@hotmail.com \\ Zulied Martínez \\ zulied@gmail.com \\ Lina Medina \\ linatheprettiest@hotmail.com \\ Lina Paola Pérez \\ linapao200@hotmail.com \\ Lizbeth Prado² \\ lucaspt5@hotmail.com
}

\author{
Hugo Cerón \\ hecerong@yahoo.es
}

Marcela Ortega Bustamante

marorbus@cable.net.co

Martha Herrera Acosta ${ }^{3}$

martha_4601@hotmail.com

\section{Resumen}

Es fundamental conocer las fortalezas y las dificultades de los estudiantes en cuanto a los procesos evaluativos que se establecen al interior de las Instituciones Educativas, además del reconocimiento de sus capacidades de conceptualización y aplicación de la teoría a sus actividades cotidianas. Por esta razón el proyecto de investigación docente realizado en el Colegio Rafael Bernal Jiménez está orientado a lograr que los estudiantes, a través del uso del aula virtual Casquete de Esfera Perforada, tengan la oportunidad de reconocer ámbitos que den cuenta de la calidad de la educación frente a los estándares básicos nacionales (pruebas censales ICFES) y estándares internacionales (programa internacional de Evaluación de Estudiantes, pruebas PISA). De forma simultánea se pretende apoyar procesos a través del uso de herramientas que les permitan familiarizarse con las Nuevas Tecnologías de la Información y la Comunicación -NTIC-, haciendo que se apropien de un conjunto de habilidades, hábitos y técnicas conducentes a fortalecer la calidad del sistema educativo.

\section{Palabras clave}

Evaluación, aula virtual, estrategia pedagógica, PISA e ICFES.

1. Proyecto de Práctica Pedagógica y Didáctica desarrollado en el IED Rafael Bernal Jiménez 2010

2. Estudiantes del Departamento de Química de la Universidad Pedagógica Nacional

3. Rector y Docentes de Biología y Química IED Rafael Bernal Jiménez 


\section{Problema}

El Proyecto de Práctica Pedagógica y Didáctica (I-II) dirigido a estudiantes de educación media y media vocacional del colegio Rafael Bernal Jiménez IED, desarrollado en el II semestre de 2009, busca fortalecer el uso de nuevas tecnologías, como el Aula Virtual, en el proceso de evaluación que se lleva a cabo en el área de Ciencias, teniendo como apoyo la implementación de evaluaciones nacionales -ICFES- e Internacionales -PISA-, como una herramienta que permita complementar el proceso evaluativo.

El interrogante que orienta la investigación de este trabajo es: ¿Es posible mejorar el proceso evaluativo en el área de Ciencias, en niveles de educación media y media vocacional en el Colegio Rafael Bernal Jiménez IED, a través de la utilización del Aula Virtual como un medio de apoyo?

\section{Objetivo}

Diseñar e implementar instrumentos y estrategias orientadas a la reflexión y búsqueda permanente del verdadero proceso evaluativo, mediante la aplicación de evaluaciones nacionales -ICFES- e internacionales -PISA- a través del Aula Virtual como mecanismo complementario a la dinámica de clase.

\section{Marco Conceptual}

Desde el punto de vista educativo, la evaluación es la acción permanente por medio de la cual se busca apreciar, estimar y emitir juicios sobre los procesos de desarrollo del alumno o sobre los procesos pedagógicos, así como sobre sus resultados, con el fin de elevar o mantener la calidad de los mismos (MEN, 1997). Las actividades de evaluación, además de ser propicias para el diagnóstico y la reflexión, se constituyen en situaciones de aprendizaje en sí mismas (SALCEDO, 1999).
Con el fin de profundizar en los temas que conciernen a la Evaluación, se recurre a la utilización de pruebas tipo PISA e ICFES con las que se busca obtener una idea bastante aproximada de los conocimientos, habilidades y aptitudes que se han acumulado a lo largo de un periodo educativo.

Específicamente, las pruebas tipo ICFES abarcan una serie de preguntas, realizadas por el Estado colombiano, que buscan informar a los estudiantes acerca de sus competencias en cada una de las áreas evaluadas, con el ánimo de apoyar los procesos de autoevaluación y mejoramiento permanente de las instituciones escolares, además de constituirse como base e instrumento para el desarrollo de investigaciones y estudios de carácter cultural, social y educativo. Esta prueba constituye tres tipos de competencias básicas, que son: la interpretativa (desarrolla las maneras de comprender gráficos, cuadros, esquemas, entre otros, dentro del marco de una situación problema), argumentativa (describe el estado, las interacciones o la dinámica de una situación problema) y propositiva (desarrolla las acciones orientadas a proponer posibles relaciones para que la situación problema suceda o pueda ocurrir).

En las pruebas tipo PISA, al igual que en las pruebas tipo ICFES, se desarrollan competencias, pero estas abarcan las áreas de competencia lectora, matemática y científica y se realizan con el fin de comparar los niveles educativos del país a nivel internacional.

En este caso, el interés se centra en las competencias de tipo científico que hacen referencia al conocimiento de la Ciencia (explicaciones científicas) y al conocimiento acerca de la Ciencia (investigación científica), dentro de las áreas de Química y Biología, en las que se busca identificar conceptos, adquirir nuevos conocimientos, explicar fenómenos y extraer conclusiones de una situación problema.

Teniendo en cuenta lo anterior, en este proyecto 
se trabaja con la implementación del Aula Virtual Casquete de Esfera Perforada. Según Horton (2000), el aula virtual es el medio en la Internet en el cual los educadores y educandos se encuentran para realizar actividades que conducen al aprendizaje; este tipo de recurso se desarrolla mediante la plataforma Moodle, que básicamente es una herramienta para diseñar cursos a través de la red. Las razones expuestas anteriormente justifican la realización de trabajos de investigación que den cuenta de la incorporación de estas tecnologías en las clases de Ciencias en general, para contribuir al aprendizaje y el desarrollo de competencias y habilidades de diferente orden en los estudiantes (Salcedo, L. E. y otros, 2008).

\section{Metodología}

El proyecto se desarrolló en cinco fases, que son:

\section{Fase de indagación y documentación}

Acerca de las pruebas tipo PISA e ICFES donde se considera su estructura, tipo de pregunta y competencia a evaluar desde las Ciencias.

\section{Fase de diseño y análisis de preguntas}

En esta fase los Docentes en Formación Inicial plantean una serie de preguntas desde el tipo de evaluaciones nacionales -ICFES- e internacionales -PISA-, según sea el caso, y de acuerdo con cada temática abordada en la dinámica de clase.

\section{Fase de indagación y documentación}

Esta fase se desarrolla a través de la página web www.casquetedeesferaperforada.net. Se lleva a cabo en varios momentos, que son:

- Adaptación, ambientación y montaje de las aulas con las temáticas correspondientes a cada grado.

- Registro de cada uno de los estudiantes en el aula virtual (asignación de usuario y contraseña).

- Aplicación de una prueba piloto, en la que se determinó la aceptación de los estudiantes frente a esta nueva herramienta didáctica.

- Una vez evaluada la prueba piloto, se procede a hacer el montaje de cada una de las pruebas para los diferentes niveles.

\section{Fase de recolección y análisis los datos}

En esta fase se recogen y analizan los datos obtenidos después de la aplicación de cada una de las pruebas.

Fase de conclusiones y sugerencias

\section{Resultados y análisis}

\begin{tabular}{|l|c|c|c|c|c|c|c|}
\hline \multicolumn{1}{|c}{ Nivel / Cursos } & 601 & 602 & 702 & 902 & 903 & 1001 & 1002 \\
\hline Número de estudiantes en el curso & 38 & 37 & 36 & 36 & 36 & 40 & 35 \\
\hline Número de estudiantes inscritos & 14 & 16 & 22 & 17 & 12 & 3 & 9 \\
\hline Número de estudiantes qe ingresan a la página & 14 & 4 & 22 & 18 & 12 & 0 & 7 \\
\hline Número de estudiantes qe realizaron la actividad & 14 & 4 & 22 & 4 & 3 & 0 & 1 \\
\hline
\end{tabular}

Tabla 1.1_Datos y variables que se analizaron. 
En la tabla anterior se observan los grados que participaron en la realización del proyecto y las variables que se consideraron para el análisis de datos. Posteriormente se muestra un análisis estadístico:
El total de estudiantes que participaron en la realización del proyecto corresponde a 258 personas.

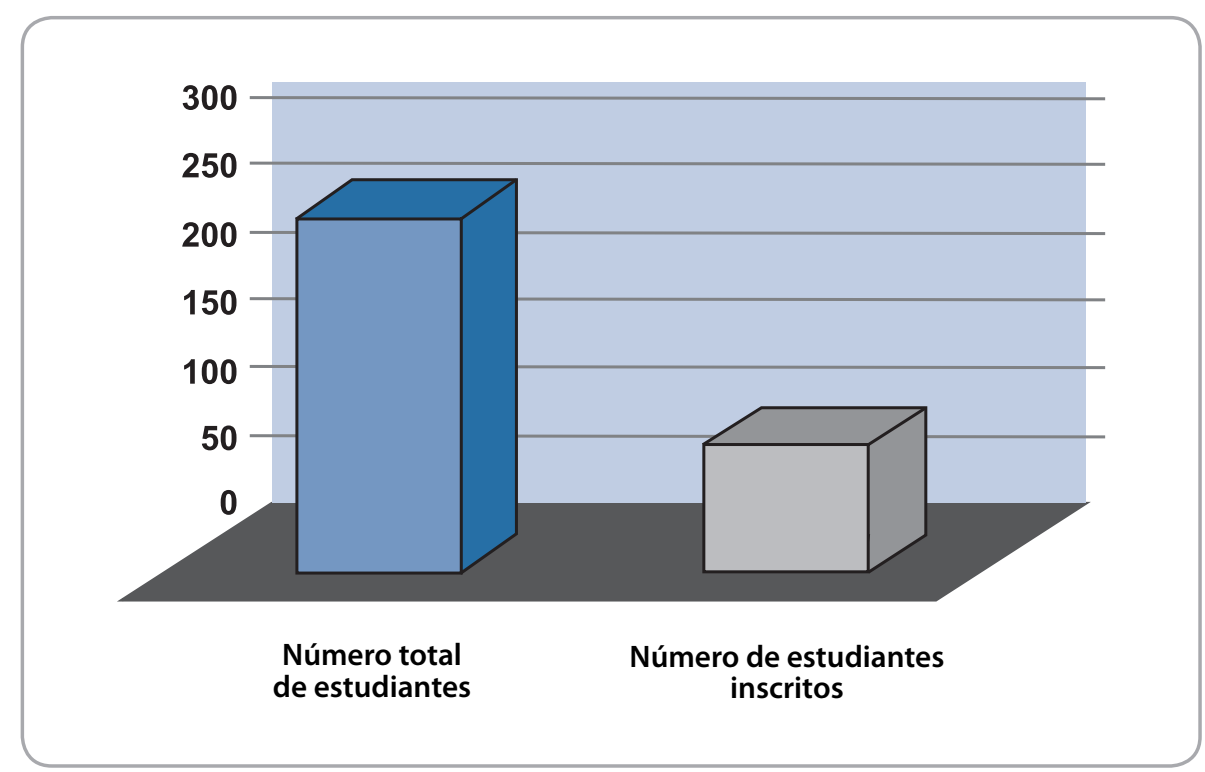

Gráfica 1.2_Del total de estudiantes participantes sólo se inscribieron al aula virtual 93, que corresponden al 36.04\%.

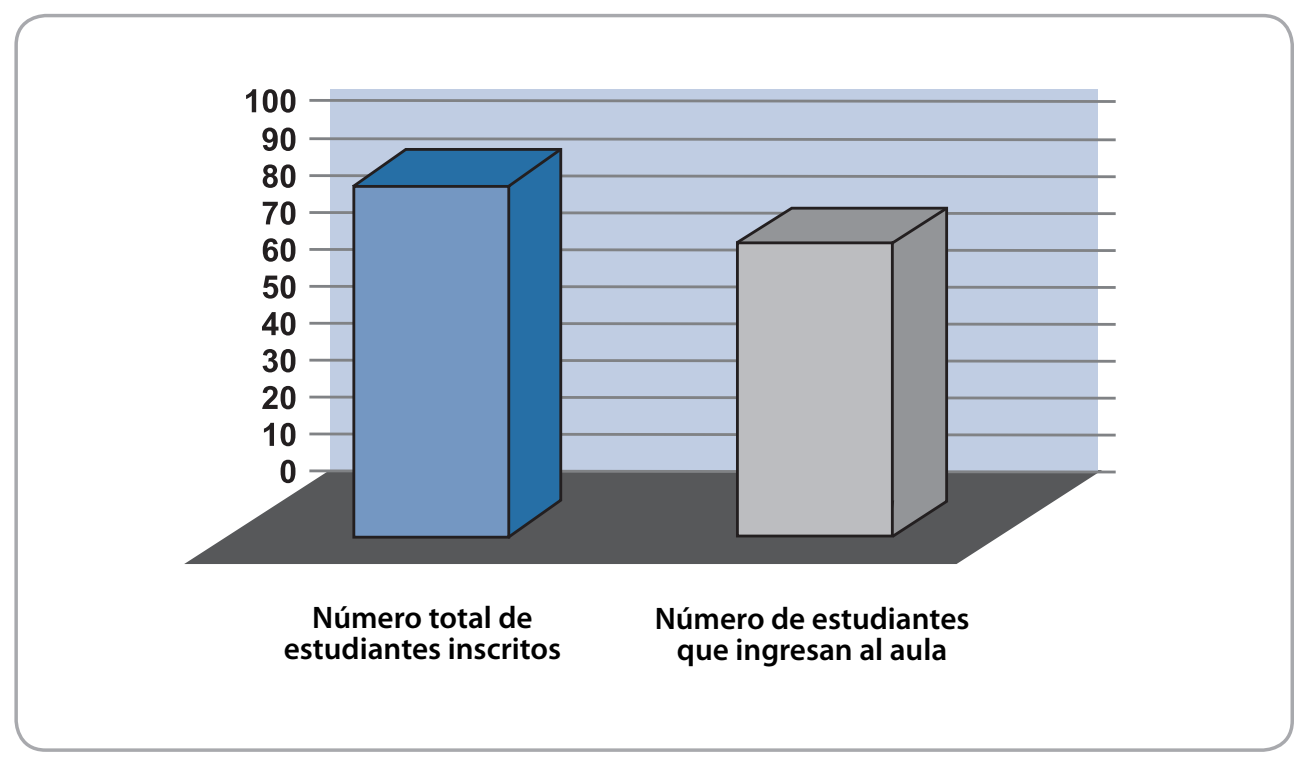

Gráfica 1.3_De los 93 estudiantes inscritos solo ingresaron a la página 77 , que corresponden al $82.79 \%$.. 


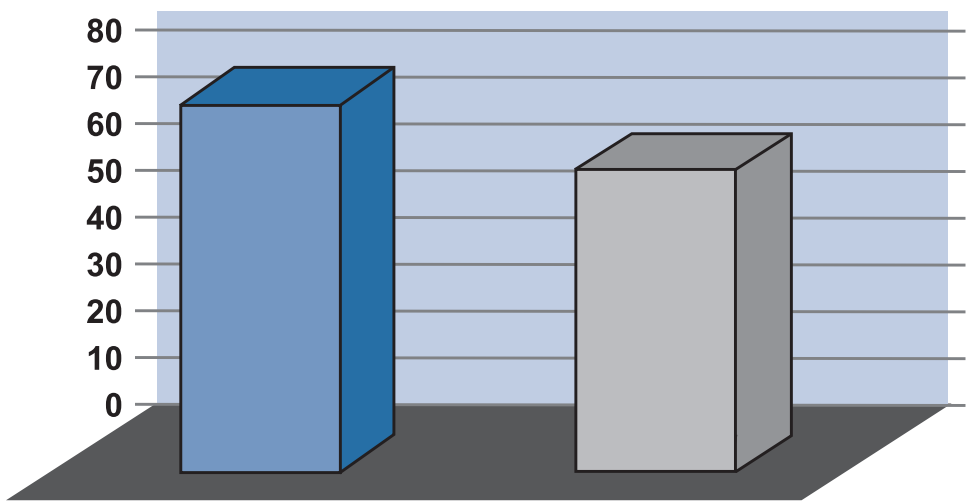

Número de estudiantes que ingresan al aula
Número de estudiantes

que resolvieron la actividad

Gráfica 1.4 _De los 77 estudiantes que ingresan a la página sólo 48 resuelven la prueba, lo que equivale a $62.33 \%$.

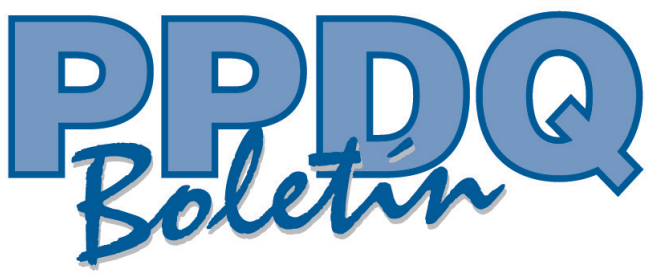

\section{Revista del Sistema de Práctica Pedagógica y Didáctica}

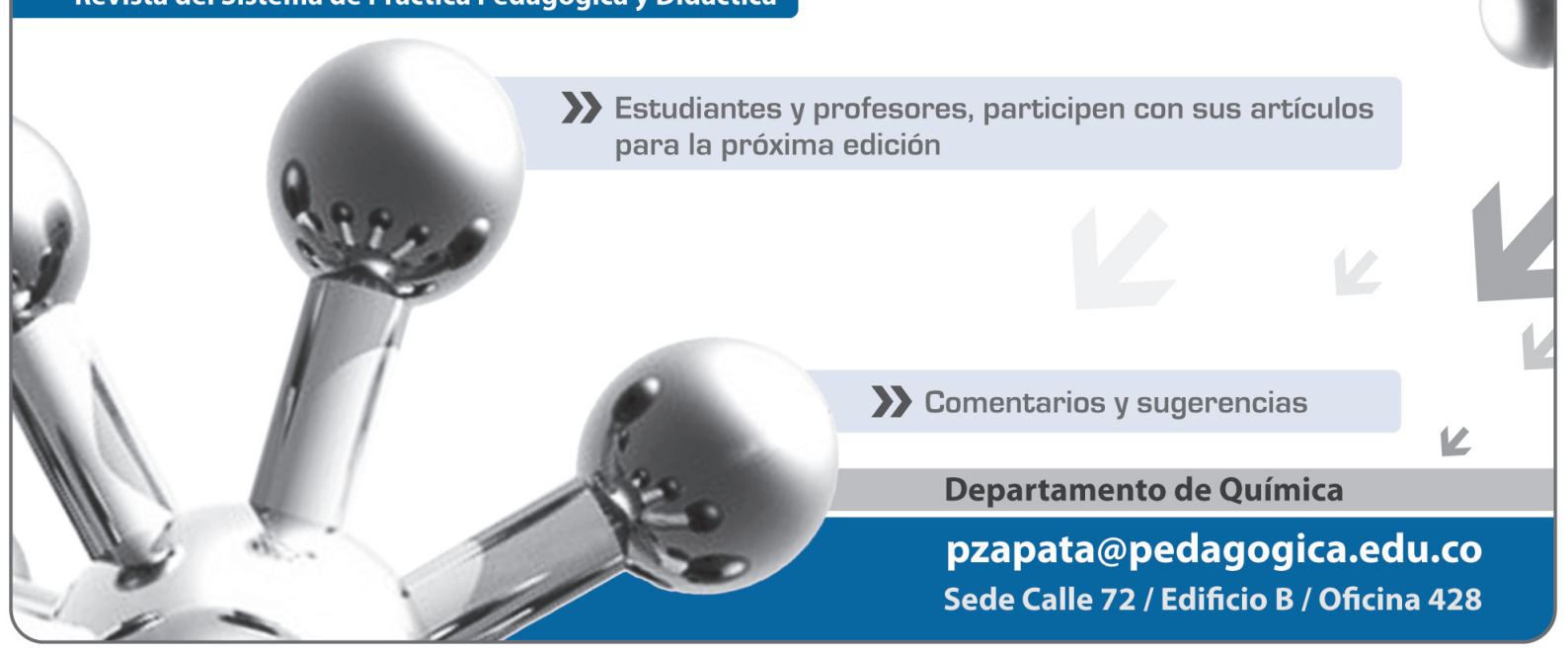


Respecto de los datos de la tabla 1.1, se observa que los estudiantes muestran dos tendencias notablemente marcadas en el desarrollo práctico del proyecto; la primera corresponde a una empatía con la interacción del aula virtual, esto con los alumnos de los grados de sexto a noveno, y la segunda una apatía y resistencia frente al mismo tema con los estudiantes de los grados décimos. Cabe resaltar que este proceso no se da tan solo por la motivación al hablarles de una nueva herramienta en el proceso de enseñanza-aprendizaje, sino que este va de la mano con un incentivo (en este caso, una nota).

Frente a las pruebas que fueron aplicadas, se tiene que las preguntas que se diseñaron desde las pruebas tipo ICFES corresponden al tipo de pregunta I, es decir, preguntas de selección múltiple con única respuesta. Estas preguntas se desarrollaron en torno a una temática, idea o problema. En algunas pruebas diseñadas varias preguntas fueron contextualizadas y, en algunos casos, compartieron un texto, una gráfica o un dibujo, a través de los cuales se aportaron elementos para la resolución de los problemas planteados en las preguntas. Las preguntas de selección múltiple con única respuesta constaron de un enunciado y cuatro opciones de repuestas designadas con letras mayúsculas $A$, B, C, y D, de las cuales solo una completa correctamente el enunciado del ítem o resuelve el problema planteado (ICFES, 2009).

Desde las pruebas tipo PISA se consideraron una serie de ejercicios que se contestan con lápiz y papel, las preguntas están agrupadas en unidades que constan de un texto introductorio, presentando una situación de la vida real, seguido de una o varias preguntas. El formato de las preguntas en este caso es cerrado, ya que estas adoptan la forma de respuesta múltiple en las que el alumno ha de escoger una sola de las opciones de respuesta presentadas (normalmente cuatro).
La implementación de este diseño de pruebas permitió fortalecer el proceso de enseñanzaaprendizaje dentro del ámbito evaluativo, pues surge un cambio en el concepto de evaluación y su papel dentro de la formación educativa. Los estudiantes comprenden que la evaluación no se limita al aula de clase, sino que es un proceso continuo que se puede enriquecer con la utilización de nuevas herramientas, en este caso el Aula Virtual.

\section{Dificultades}

Dentro de la realización del proyecto se presentan varias dificultades en la implementación del Aula Virtual, entre las que se encuentra que muchos de los estudiantes no cuentan con el acceso a la red, bien sea por falta de recursos o por falta de conocimiento sobre su uso.

Frente a esta problemática se hace necesario realizar un proceso de reconocimiento, gracias a dos equipos que se encuentran en la sede $A$ del colegio y que cuentan con los programas y la conexión a la red.

\section{Sugerencias}

Es fundamental que dentro de los colegios se visualice a las Nuevas Tecnologías de la Información y la Comunicación, y específicamente al uso del Internet, como herramientas transversales, que pueden ser utilizadas por todos los maestros desde las diferentes áreas para complementar el proceso educativo.

Es necesario que los maestros en ejercicio primero se fundamenten acerca de estos temas y se apropien de los mismos, con el objetivo de dinamizar y enriquecer los procesos de formación. 


\section{Conclusiones}

- Al diseñar e implementar instrumentos de evaluación tipo ICFES y PISA bajo las Nuevas Tecnologías de la Información y la Comunicación -NTIC- se logra que los estudiantes y docentes del plantel educativo conozcan dichas herramientas, a nivel de educación, como ayuda en el proceso de enseñanzaaprendizaje.

- A través de la implementación y montaje de los diferentes tipos de preguntas se logra que los estudiantes se familiaricen con las pruebas tipo ICFES y PISA, así como que reconozcan la estructura de las preguntas.

- Se reflexiona sobre nuevas modalidades para llevar un proceso educativo y evaluativo continuo, en el que se aporten elementos innovadores.

\section{Bibliografía}

Salcedo, L. E., Villareal, M., Zapata, P., Colmenares, E., García, M., Moreno, P. (2008). Tecnologías de la información y la comunicación en educación química. Bogotá: Colciencias, Universidad Pedagógica Nacional.

Salcedo, L. E., Villareal, M. (1999). Educación y Pedagogía. (11) 23-24. Universidad de Antioquia.

Horton, W. (2000) . Designing Web-Based Training. New York: John Wiley.

Brown L., Lemay H. E. (2004). Química la ciencia central. Novena edición. México: Pearson Educación, Prentice Hall.

[ㄷ] Daub, W., Seese, W. (2005). Basic Chemistry. Séptima edición. Edición Pearson. 


\section{Anexo}

\section{DISEÑO DE PREGUNTA TIPO PISA PARA EL GRADO 702}

\section{La ciudad, un ser humano gigante}

Si observamos de manera detenida el funcionamiento de una ciudad, encontramos muchas similitudes con el funcionamiento interno del ser humano. Por ejemplo: El sistema de transporte masivo se asemeja con nuestro sistema circulatorio, más cuando es de color rojo y lo podríamos llamar Transoxilenio, se mueve a toda hora cargado de personas que recorren la ciudad de extremo a extremo para ir a cumplir con todas sus labores diarias.

La ciudad cuenta con varias empresas encargadas del aseo que, con un personal encargado y camiones, recogen por todos los rincones los desechos producidos por las fábricas, supermercados, hogares, etc. Esta labor es muy afín con la función que realizan el sistema excretor y el sistema inmunológico.

Las grandes extensiones de zonas verdes con árboles y plantas de los parques de nuestra ciudad ayudan a oxigenar y purificar el aire, que tan contaminado se encuentra por la emisión de gases producida por los automóviles, fábricas, quema de residuos, etc. Esta función la realizaría nuestro sistema respiratorio, proporcionándonos oxígeno y eliminando el gas carbónico de nuestro cuerpo.

También está el sistema de acueducto y alcantarillado que se encarga de suministrar el agua potable a la comunidad y de recoger los desechos que llegan de las aguas lluvias y domésticas. De igual manera, esta función la realiza nuestro sistema digestivo, suministrándonos nutrientes y eliminando los desechos.

En conclusión, la ciudad se puede considerar como un ser humano que respira, que tiene necesidades y que requiere de cuidados. En nuestras manos está que este ser no muera y que tenga buena salud.

Pregunta de selección múltiple con ÚNICA respuesta. Tipo I

Pregunta 1. El sistema circulatorio es comparado con el transporte masivo porque:

a) Se mueve a altas velocidades, por las calles y avenidas principales.

b) Las personas lo utilizan todos los días del año para ir a sus trabajos.

c) Sirve de transporte por las vías o redes y recorren toda la ciudad.

d) Tiene un color rojo que es parecido al color de la sangre humana. 
Pregunta 2. Una acción de tantas que podríamos realizar los seres humanos para cuidar al sistema respiratorio de nuestra ciudad es:
a) Utilizar en forma adecuada el sistema de transporte masivo.
b) Utilizar en forma adecuada los electrodomésticos.
c) Evitar arrojar basuras a las calles y sembrar más árboles.
d) Impedir la quema de basuras y sembrar árboles.

Pregunta 3. Los órganos que se encargan de funciones similares a las realizadas por las empresas de acueducto y alcantarillado son:
a) El estómago, los bronquios y alvéolos.
b) Las arterias, las venas y el hígado.
c) La vejiga y los riñones.
d) El estómago y los intestinos.

Pregunta 4. El sistema inmunológico tiene una función que puede ser comparada con la policía, porque:
a) Siempre está lista para servir.
b) Atrapa a quienes hacen años.
c) Protege contra cualquier ataque.
d) Recorre toda la ciudad.

\section{Diseño de pregunta tipo ICFES para los grados décimo}

\section{Preguntas:}

Las sales haloideas o hidrosales se obtienen por la reacción de un ácido hidrácido y una base. Se caracterizan porque en su estructura no hay átomos de oxígeno; se clasifican, según tengan o no átomos de hidrógeno, con fórmulas generales MHNm y MNm, respectivamente. Teniendo en cuenta lo anterior, el nombre correcto para estos subgrupos será:
a) Hidrosales neutras e hidrosales simples.
b) Hidrosales básicas e hidrosales ácidas.
c) Hidrosales ácidas e hidrosales neutras.
d) Hidrosales dobles e hidrosales simples

Componente: Aspectos analíticos de las sustancias.

Competencia a desarrollar: Interpretativa. 
Las sales se forman por la reacción entre un ácido y una base, en donde la especie catiónica proviene de la base y la aniónica del ácido; teniendo en cuenta lo anterior y sabiendo que una sal proviene de la reacción del ácido sulfúrico con hidróxido cuproso, indique la fórmula y el nombre que corresponden a la sal formada

a) $\mathrm{Cu} 2\left(\mathrm{SO}_{4}\right)$ sulfato cuproso.

b) CuSO4 sulfato cúprico.

c) $\mathrm{CuSO}_{3}$ sulfito cúprico.

d) $\mathrm{CuHSO}_{4}$ sulfato ácido cuproso.

Componente: Aspectos físico químicos de la sustancias.

Competencia a desarrollar: Argumentativa. 